TM-1660

\title{
Investigation of the Mechanical Properties of Superconducting Coils
}

\author{
F. W. Markley and J. S. Kerby \\ Fermi National Accelerator Laboratory \\ P.O. Box 500 \\ Batavia, Illinois 60510
}

March 1990

* Submitted to the 1990 International Industrial Symposium on the Super Collider (IISSC), Miami Beach, Florida, March 14-16, 1990. 
INVESTIGATION OF THE MECHANICAL

PROPERTIES OF SUPERCONDUCTING COILS

\author{
F.W. Markley and J.S. Kerby \\ Fermi National Accelerator Laboratory* \\ P.O. Box 500 \\ Batavia, Illinois 60510
}

\begin{abstract}
This paper presents data on 3 of the important mechanical properties of SSC type superconducting coils. The measured properties are: 1) The azimuthal elastic modulus of the coil samples made for the stress relaxation tests. 2) The rate of stress - relaxation cf collared SSC outer coils molded to different sizes and 3 ) The pressures that various insulations can withstand during molding or collaring before turn-to-turn shorts develop. Additional data on these and other properties are available but omitted here because of space limitations.
\end{abstract}

\title{
SAMPLE PREPARATION
}

Coil samples for stress relaxation and modulus testing were molded in $3^{\prime \prime}$ long molds. During the cure, a shim was placed between the top of the fixture and the hydraulic ram loading the coil. This shim determined the cured size of the coil. The hydraulic pressure was continuously adjusted by hand so that this shim could just be moved back and forth between the fixture and the ram. Thus we were able to monitor the force required to just keep the coil at the desired size during the cure. When an additional shim is added to this shim to make an oversize sample, it is identified as being made with a negative shim. When an additional shim is placed on top of the coil during cure to make an undersized sample, it is identified as being made with a positive shim.

\section{COIL MODULUS}

We measured the modulus of all the samples and found the stress strain curve to become reasonably linear near the high stress end. All samples gave a modulus of $1.4 \times 10^{+6} \pm .4 \times 10^{+6}$ psi, regardless of the size to which they were molded.

\section{STRESS RELAXATION}

Figure 1 is a drawing of the stress relaxation fixtures used (there are three). The solid stee] blocks on either side of the load cells are spacers to allow the use of several different load cells with different dimensions. The curved block supporting the coil has a slightly larger radius than the block used to cure the coil because now the ground insulation has been added. The notch just above the open space holds a steel spacer with the coil I.D. that keeps the coil in place during assembly of the coil into the fixture.

We decided to build the fixtures to have the same total compliance that is expected for the SSC collars. Robert Wands had done a finite element analysis for the collars that predicted a compliance of $.45 \times 10^{-6}$ inches per pound in the azimuthal direction. One of the load cell and fixture combinations turned out to have a larger compliance than planned. The compliances of the

"Operated by Universities Research Association under contract with the U.S. Department of Energy 


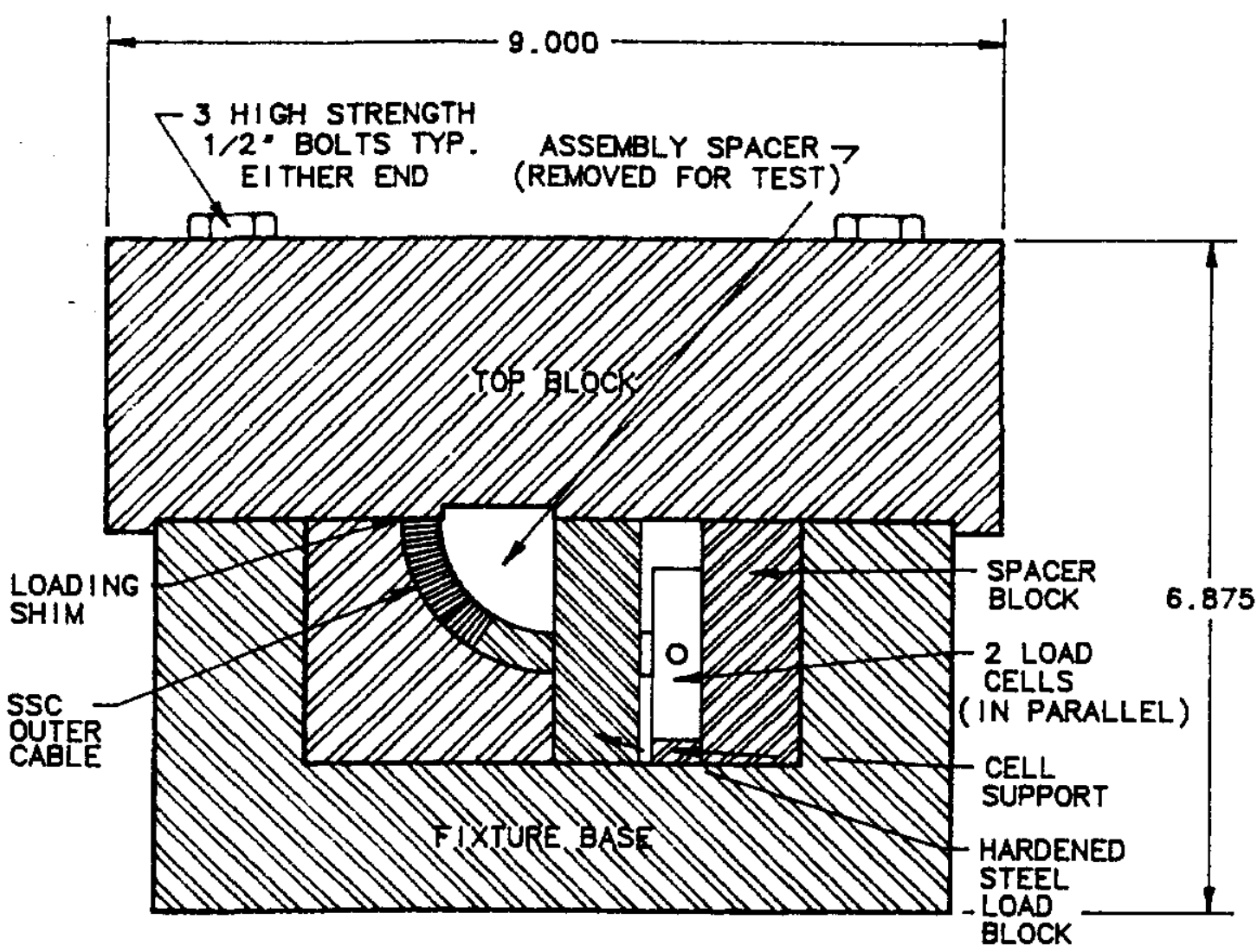

Fig. 1. Stress Relaxation Fixture

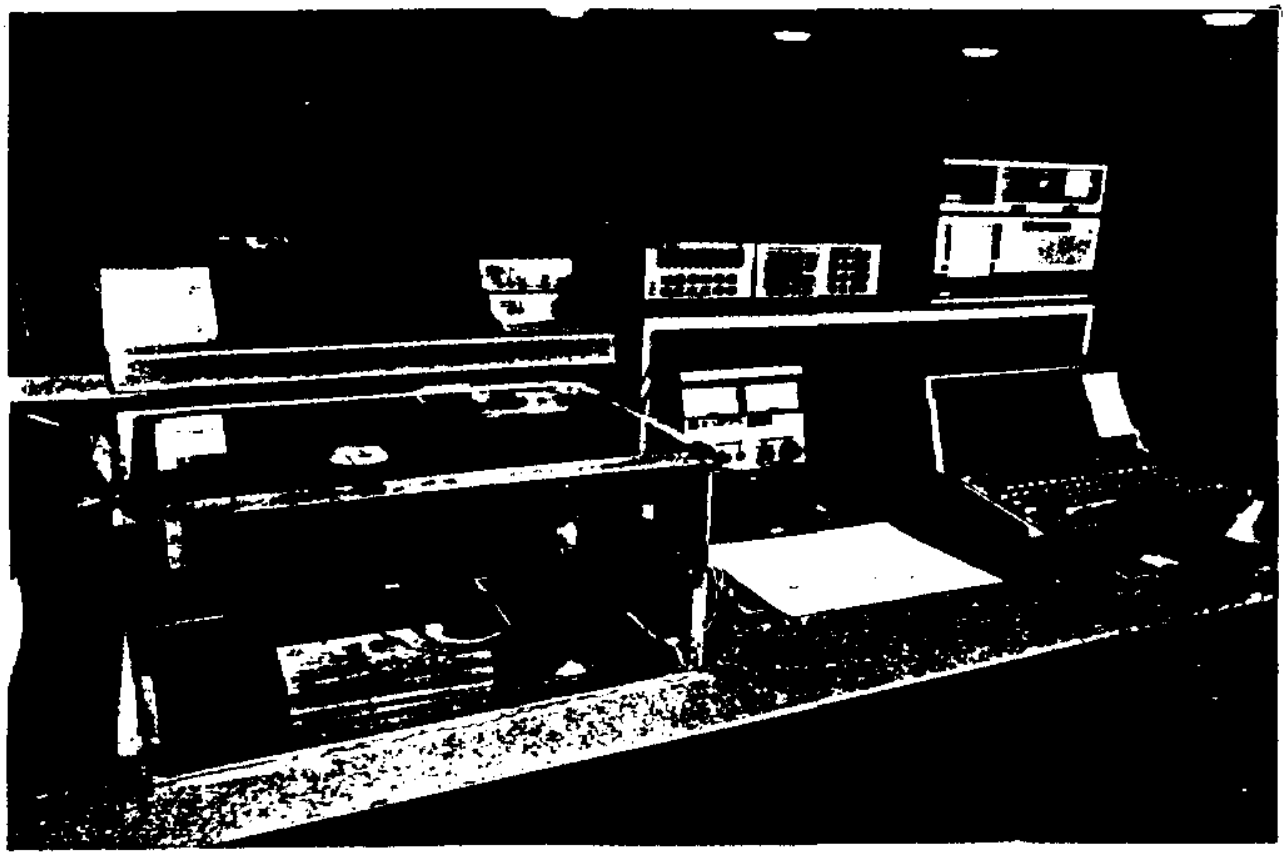

F1g. 2. Stress Relaxation Setup 
load cell-fixture combinations were measured by substituting a solid steel block and a series of shims for the coil and measuring the load cell output when the upper half of the fixture was tightened down. The compliance was then taken as the slope of the line on the plot of shim thickness versus load. Two fixtures were used to take the data reported here and one had a compliance of $1.210^{-6}$ inches per pound and was used for samples numbered $4,7,11$, and 13,13R. The other had a compliance of $.510^{-6}$ inches per pound and was used for samples numbered 8,12 , $14,15,16$, and 19. These compliances are only used in the fixture corrected stress relaxation calculation.

Figure 2 is a photo of the entire creep apparatus. The fixture is enclosed in a plastic box with an electric heater and a fan. The heater is controlled by a PID controller and a thermocouple in the air downstream from the fan. The air temperature is controlled to $90^{\circ} \mathrm{F}, \pm .2^{\circ}$. The coil temperature is measured with a thermocouple in the fixture near the top of the coil.

The two load cells in each fixture are powered in series by a very stable HP6186C constant current power supply and their output voltages are measured with an HP3457A $61 / 2$ digit voltmeter. The voltmeter has an IEEE488 buss and is computer controlled to measure the voltages every 4 minutes and record them on magnetic discs where they are later converted to load.

Figure 3 is the raw load versus time for 9 coil samples. The shims used to mold these samples are as follows:

$\begin{array}{lccr}\begin{array}{l}\text { Coil } \\ \text { Sample }\end{array} & \begin{array}{l}\text { Mold Shim } \\ \text { Thickness }\end{array} & \begin{array}{l}\text { Measuring Shim } \\ \text { Thickness }\end{array} & \begin{array}{c}\text { Initial PSI } \\ \text { Load }\end{array} \\ \# 13 & -.008^{\prime \prime} & -.020^{\prime \prime} & 3020 \\ \# 13 R & -.008^{\prime \prime} & +.007^{\prime \prime} & 12718 \\ \# 14 & -.008^{\prime \prime} & +.018^{\prime \prime} & 8511 \\ \# 4 & .000^{\prime \prime} & .000^{\prime \prime} & 2469 \\ \# 7 & .000^{\prime \prime} & .000^{\prime \prime} & 6313 \\ \# 8 & .000^{\prime \prime} & .000^{\prime \prime} & 10105 \\ \# 11 & +.008^{\prime \prime} & ? & 5790 \\ \# 12 & +.008^{\prime \prime} & +.029^{\prime \prime} & 12100 \\ \# 15 & +.016^{\prime \prime} & +.025^{\prime \prime} & 9250 \\ \# 16 & +.024^{\prime \prime} & +.026^{\prime \prime} & 10032 \\ \# 19 & +.040^{\prime \prime} & +.026^{\prime \prime} & 8918\end{array}$

The loads given for samples $13 R$ and 15 are the first measured points, the rest are extrapolated to 0 times. At first we tried to mold and measure a coil using the same shim thickness, but the loads were too low for accurate measurement so we began using larger measuring shims (similar to collaring to a smaller size) and trying to start at the same load. The data in Figure 3 are very hard to compare because of the large load range and the small variation. Obviously some kind of normalization is needed.

We can justify the normalization in the following way. The stress relaxation modulus is $G(t)=\sigma(t) / \varepsilon$ and the unrelaxed modulus (the value at $t=0)$ is $G_{0}=\left(\sigma_{0}\right) / \varepsilon_{0}$. Remembering that strain is nearly constant; i.e. $\varepsilon_{0}=\varepsilon$, we can combine these equations to get $G(t)=G_{0} *\left(\sigma(t) / \sigma_{0}\right)$, and divide by the area for $G(t)=G_{0} *\left(1 / 1_{0}\right)$. Dividing our loads by the initial load will nicely normalize our data to a value of $G_{0}$. Since the actual value of $G_{O}$ is not important to comparing data, we choose to use for it's value the ordinary modulus we have previously measured. Figure 4 is a graph of the data normalized in this way, using $E=1.1 \times 10^{+6} \mathrm{psi}$.

The most unusual feature of the data is that samples 12 and 13 do not agree with the rest. In fact, sample 11 is also unusual in that it seems to be decreasing at a nearly linear rate. These differences are not a function of the shim size used in the molding of the samples since 12 was made with a +.008 inch shim and 13 was made with a -.008 inch shim. We have remeasured sample 13 after a long relaxation time and got very different results that agree with the majority of the other samples. This data is shown on the graph as sample 13R. The straight line portion 
4

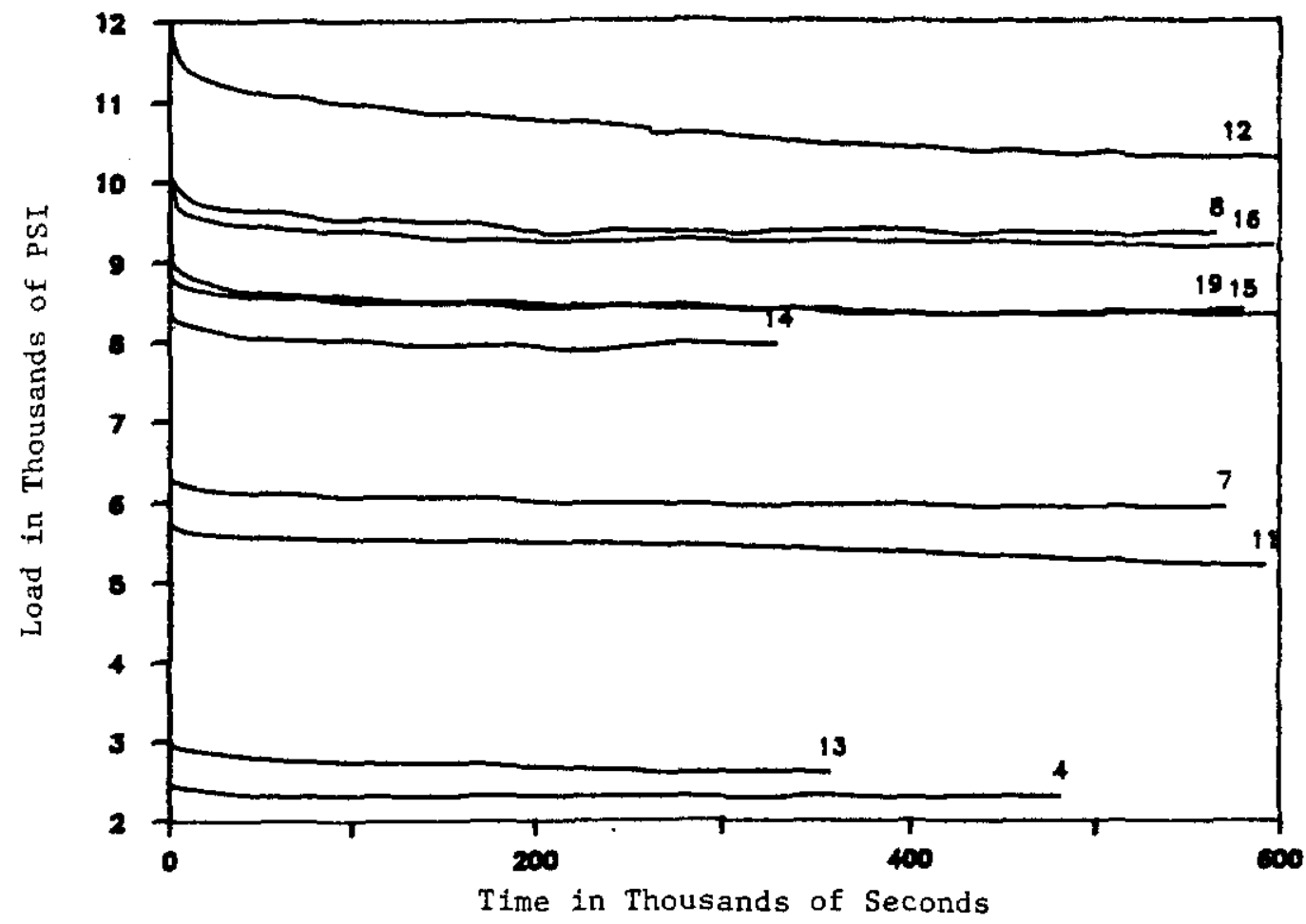

Fig. 3. SSC outer coil test load in psi as a function of time for samples molded to various final sizes.

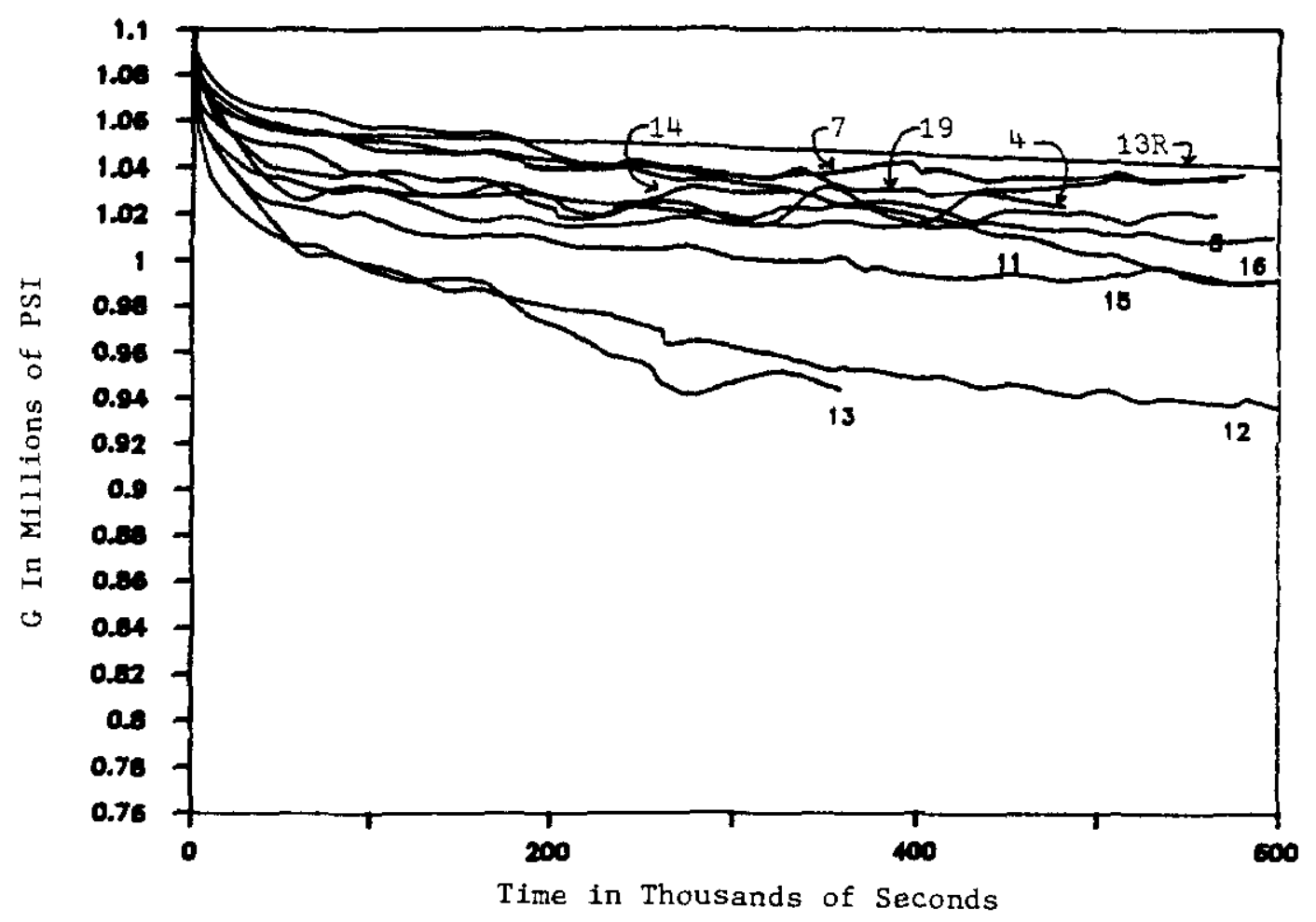

Fig. 4. Stress relaxation modulus as a function of time (assuming an unrelaxed modulus of $1.1 \times 10^{+6}$ ). 
of $13 \mathrm{R}$ is due to a data acquisition failure and the line connects to additional points off the scale of the graph. We would like to remeasure sample 12 also.

The second obvious feature of the data is that most of the samples relax at about the same rate regardless of the shim thickness used in their molding. It has been suggested that the Kapton part of the insulation system would undergo stress relaxation at a rapid rate during the high temperature molding cycle, and would recover from that history at a lower temperature and therefore, a slower rate. This stress history might then affect the relaxation rate after collaring. No such effect is apparent. There are too many experimental variables to conclude much beyond the obvious that it is not easy to change collared coil visoelastic behavior by modifying coil molding parameters.

We were concerned that the 11 different relaxation curves were obtained from two different fixtures with different compliances and one was somewhat different from the predicted compliance of the SSC collars. We have, therefore, computed a correction to the data that will approximately remove the effect of compliance. The stress relaxation modulus $G=\sigma(t) / \varepsilon_{0}$ which we can rewrite as $G=(1(t) / A)(L / \Delta L)$ where $1(t)$ is the load, $A$ the area, $L$ the length, and $\Delta L$ the change in length. Now as the load on the sample decreases with time, the load on the fixture decreases accordingly and the fixture opening decreases imposing an additional deformation on the sample. This additional deformation can be written as $\Delta \mathrm{L}=\Delta \mathrm{L}_{\mathrm{o}}+\mathrm{c}\left(1_{\mathrm{o}}-1\right)$, where $\mathrm{c}$ is the compliance of the fixture and $\Delta \mathrm{L}_{\mathrm{o}}$ is the deformation at time zero, and $\mathrm{l}_{\mathrm{o}}$ is the load at time zero. Substituting this value of $\Delta \mathrm{L}$ into the equation for $\mathrm{G}$ gives us $\mathrm{G}=(1 / \mathrm{A})\left(\mathrm{L} /\left(\Delta \mathrm{L}_{\mathrm{o}}+\mathrm{c}\left(\mathrm{l}_{\mathrm{O}}-1\right)\right)\right.$. If we note that at time zero $G$ is the unrelaxed modulus $G_{0}=\left(1_{0} / A\right) /\left(L / \Delta L_{0}\right)$, we can solve for $\Delta \mathrm{L}_{0}$ and substitute into the equation for $G$. Rearranging terms $G=\left(1 / 1_{0}\right)^{*} G_{0}^{*}\left(1 /\left(1+\left(c^{*} A^{*} G_{0} / L\right)\left(1-1 / l_{0}\right)\right)\right.$. Note that when the fixture is perfectly rigid, the compliance is 0 and the expression for $G$ reduces to the same expression we used to normalize our data. Also note that for $c>0$, the fractional term is less than 1 , which implies that for any given value of $G$ the load $I$ must be larger than it would be for the $c=0$ case. This is to say that stress relaxation in any real fixture occurs more slowly than for a hypothetical case of constant strain. Figure 5 shows the data modified in this way to correct for the fixture compliance. The effect is seen to be an increase in the spread of the data which is unfortunate even though it does make a sample 12 look less far off the median.

Figure 6 shows some very long term data. Texts on visoelastic theory $y^{1,2}$ state that crosslinked plastics like epoxies should relax to some constant value of stress while thermoplastics like Kapton may continue to relax indefinitely due to an actual irreversible flow of the material. The long term data shows a continuing relaxation. It can easily be seen that if the data had stopped at some unfortunate earlier period, it might have been interpreted as having reached a limit. This illustrates just one of the difficulties in these measurements.

We can look back at an earlier paper ${ }^{3}$ where we measured relaxation rates on straight stacks of Tevatron cable and find a remarkable agreement considering the difference between the two experiments. Since the primary stress relaxing element in both experiments is assumed to be the Kapton, we are encouraged to use the time-temperature shift factor measured in the earlier case as the best available data until it can be remeasured with actual SSC coils.

\section{INSULATION BREAKDOWN}

The maximum pressure that insulated SSC cable can withstand before electrical breakdown occurs turns out to be a surprisingly complicated subject. It is most important because of the pressure that must be applied both in molding a coil and in collaring the final coils. We have not considered the insulating value of the helium, but instead have applied a turn-to-turn voltage of 2 kilovolts which is sufficient to cause breakdown whenever the plastic insulation has ruptured.

We have found three different modes of failure of the Kapton insulation. The first mode is found whenever there is a flaw in the cable construction. Under the microscope we have found cables with strands of varying diameters which cause irregular decreases in the flat area on the surface of each strand which area actually supports the applied load. We have found strands with distinct bumps on the flat surface especially near the cable edges. These bumps can pierce the insulation much like a foreign inclusion would. We have also found cables where some of the 


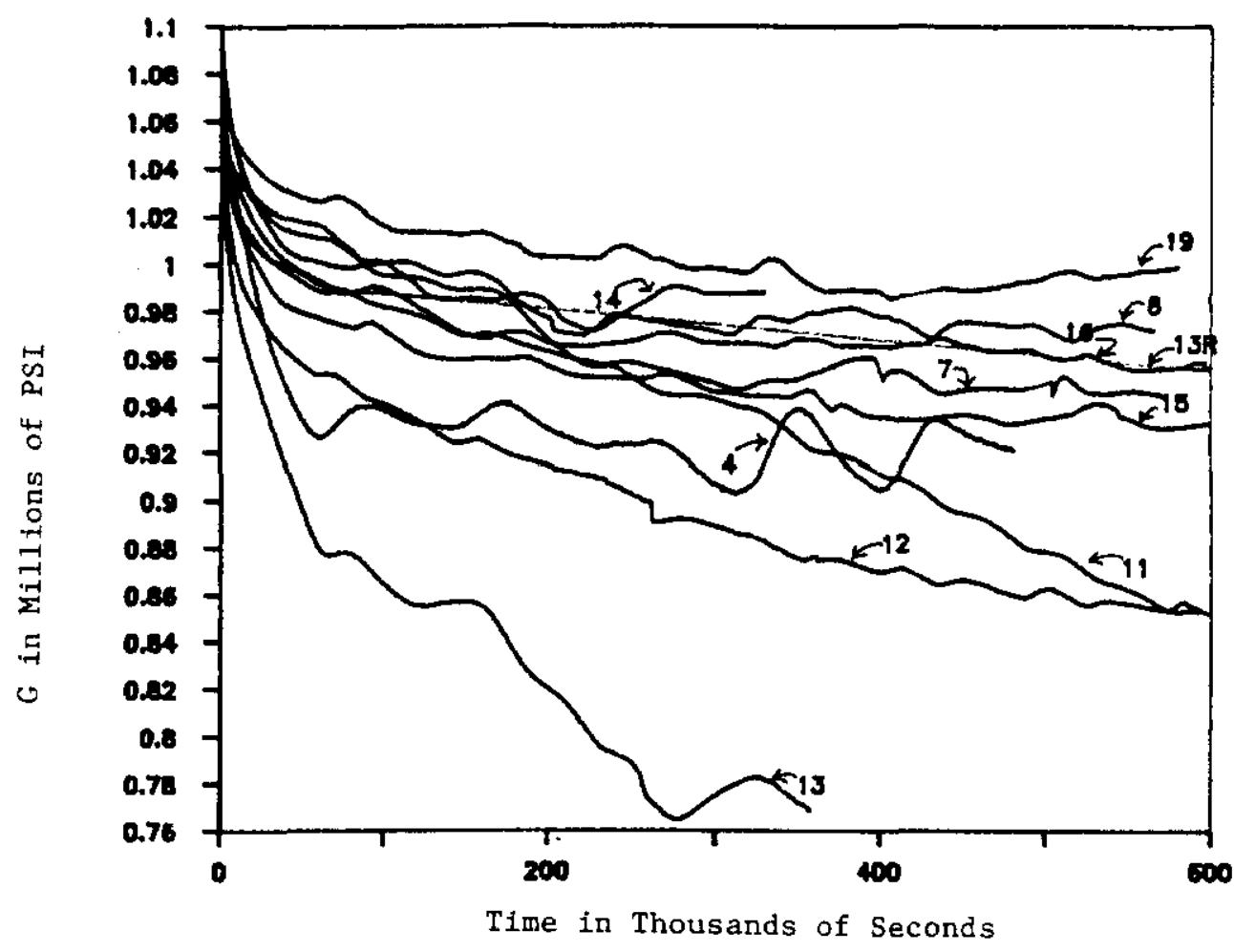

Fig. 5. Stress relaxation modulus corrected for fixture compliance.

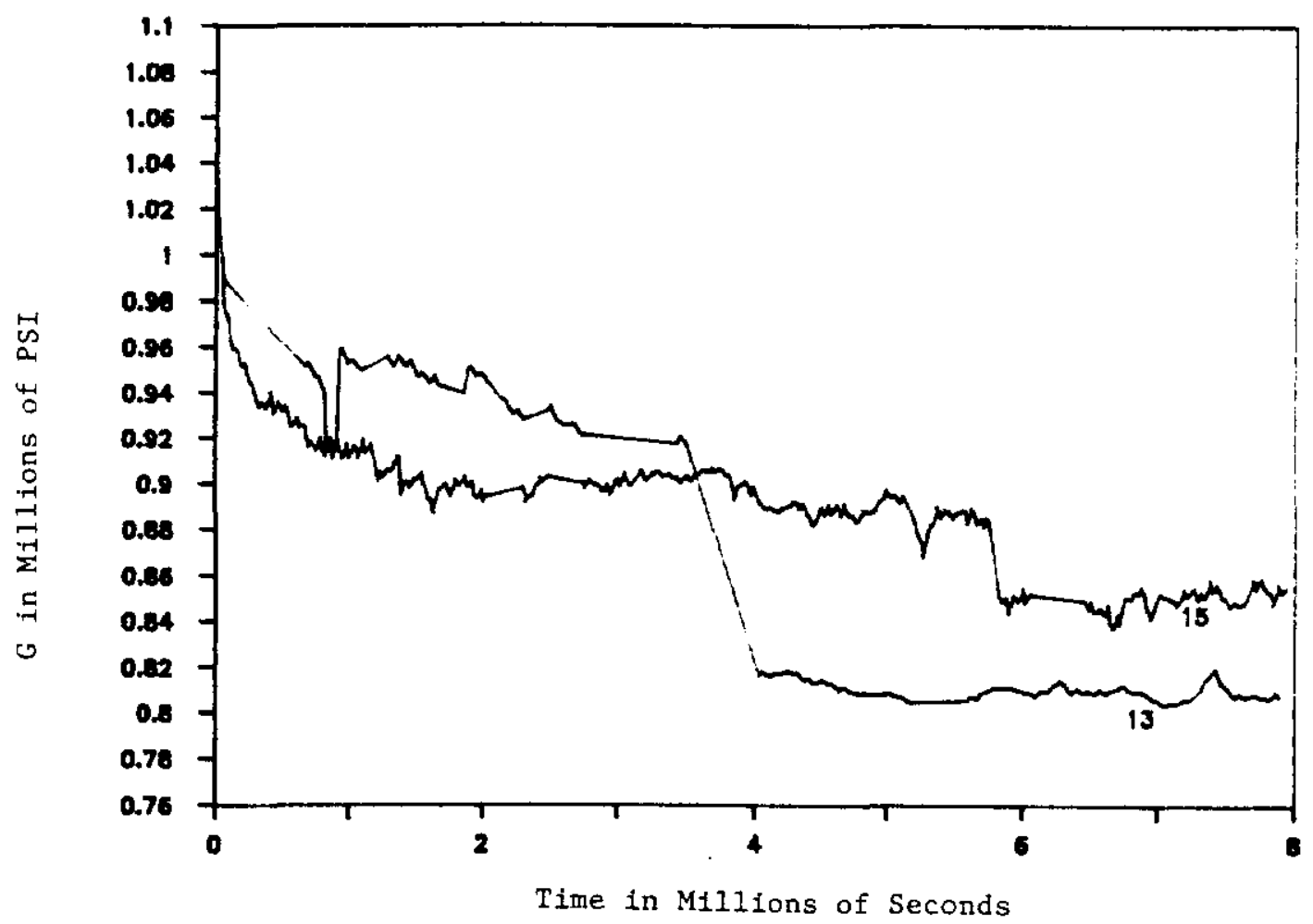

Fig. 6. Long term stress relaxation modulus. 
strands have badly pitted surfaces. These pits look as if the copper had been torn out of the flattened cable surface by a galling contact with the cable flattening dye. The Kapton appears to extrude into these holes and in doing so, thins at the hole edges where ruptures then occur. We have seen many instances of cable strands with sharp protrusions on the edges of the flattened regions. Others have reported seeing these protrusions so bad that they break off the strand edges as long copper hairs. All of these flaws can cause failure of the insulation at low values of pressure. It will be vital to have a good inspection system for incoming cable to detect flaws and reject cable. We have tried to correct flawed cable by sanding and/or polishing the cable surfaces, but have not been successful. The complex behavior of these kinds of flaws is illustrated by the tests in which we took good cable and created flaws by sanding and scratching the surface. When insulated and tested, only surprisingly small decreases in the pressure to breakdown were found. Figure 7 shows the breakdown pressures of 4 different cables. Of the two SSC cables, the one with the lower average breakdown pressure was found to have strands with the pitted surfaces mentioned previously.

The second mode of failure is a series of cuts through the Kapton where the cuts are found along and aligned with the edges of the strand flat surfaces. They were found on both cables at a mating surface. Several such cuts are usually found even though the charred indications of an electrical arc are only found at a single spot. Obviously, a failure must be located at the intersection of an upper and lower strand flat surface edge. We consider these failures to be the predominate type on high quality cable.

The third failure type is found whenever extra layers of Kapton are used or the insulation system otherwise modified to give rise to failures at a much higher value of pressure. In these cases, the higher pressure causes the cable to spread in the width direction which tears the Kapton apart. If the cable is restrained from this spreading, much larger pressures can be reached before insulation failure. In the present design, the outer coil might be so restrained by the presence of the inner coil, but the inner coil is not restrained. It is conceivable to design a magnet where the inner coil is also restrained by filling the space between the beam tube and the inner coil with a material capable of transferring stress from the coil to the beam tube. We have measured the residual increase in width of several types of cable as a function of the pressure to which we have exposed them. There is a small residual width increase at very low pressures which does not change much as the pressure is increased. At a pressure of around $50 \mathrm{Kpsi}$, the residual width begins to increase in a nearly linear manner with pressure. This pressure is the transition point to insulation failures of the third type.

In Figure 7, the Staybright cable is not only much better than the other cable types, but it is visually different in having a much larger flat area on the top of each strand. The cable must have been flattened much more than usual in the final rolling. This prompted us to make a measurement under the microscope of the total flat area found in the Staybright cable and the two SSC cables. The flat area of a single strand was measured very carefully with a microscope and video camera measuring system. For ease of calculation, this area was used to find the width of a strand having the same area, but a uniform width. We then calculated the area of the intersections of the upper and lower cable strands of this width. This gives a good approximation of the true area of the cable actually supporting the applied load. Using the calculated areas, the pressure at breakdown was found to be $113 \mathrm{Kpsi}$ for Staybright, $156 \mathrm{Kpsi}$ for SSC22-0006, and $125 \mathrm{Kpsi}$ for SSC23-384b. Thus the apparent superiority of the Staybright cable can be completely explained by it's increased area, but the superior cable SSC22-0006 compared to SSC23-384b must still be explained by the damaged surface of the latter cable. The data of Figure 8 were taken using a single 1/2-lapped layer of Kapton and two cables stacked narrow edge next to wide edge and loaded between parallel steel plates (the Fermi fixture). No epoxy fiberglass was used.

In Figure 8, we compare the breakdown pressure of the Kapton only insulation with data obtained when epoxy fiberglass is added in both the uncured and cured condition. Also shown are data from both the Fermi style fixture and a fixture originated by Brookhaven. The Brookhaven fixture used a shorter length of cable in a round die shaped to take two cables stacked narrow edge to narrow edge and constraining those cables on their edges. We have built fixtures of this type to take both inner cable pairs and outer cable pairs. Unless otherwise noted, our data was taken with outer cable. 


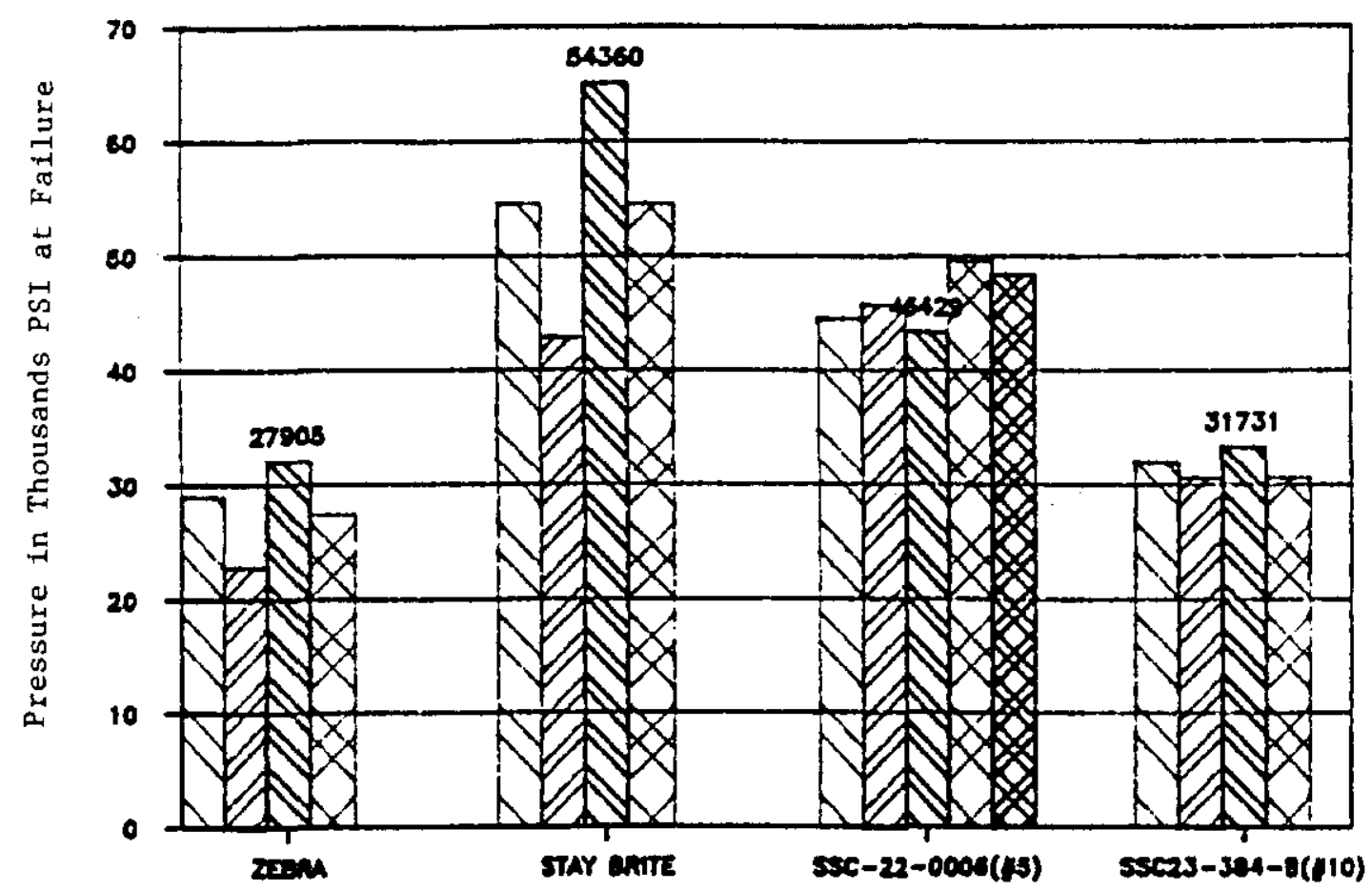

Fig. 7. Pressure to cause electrical breakdown of 1 layer of $1 / 2$ lapped Kapton insulation on various superconducting cables.

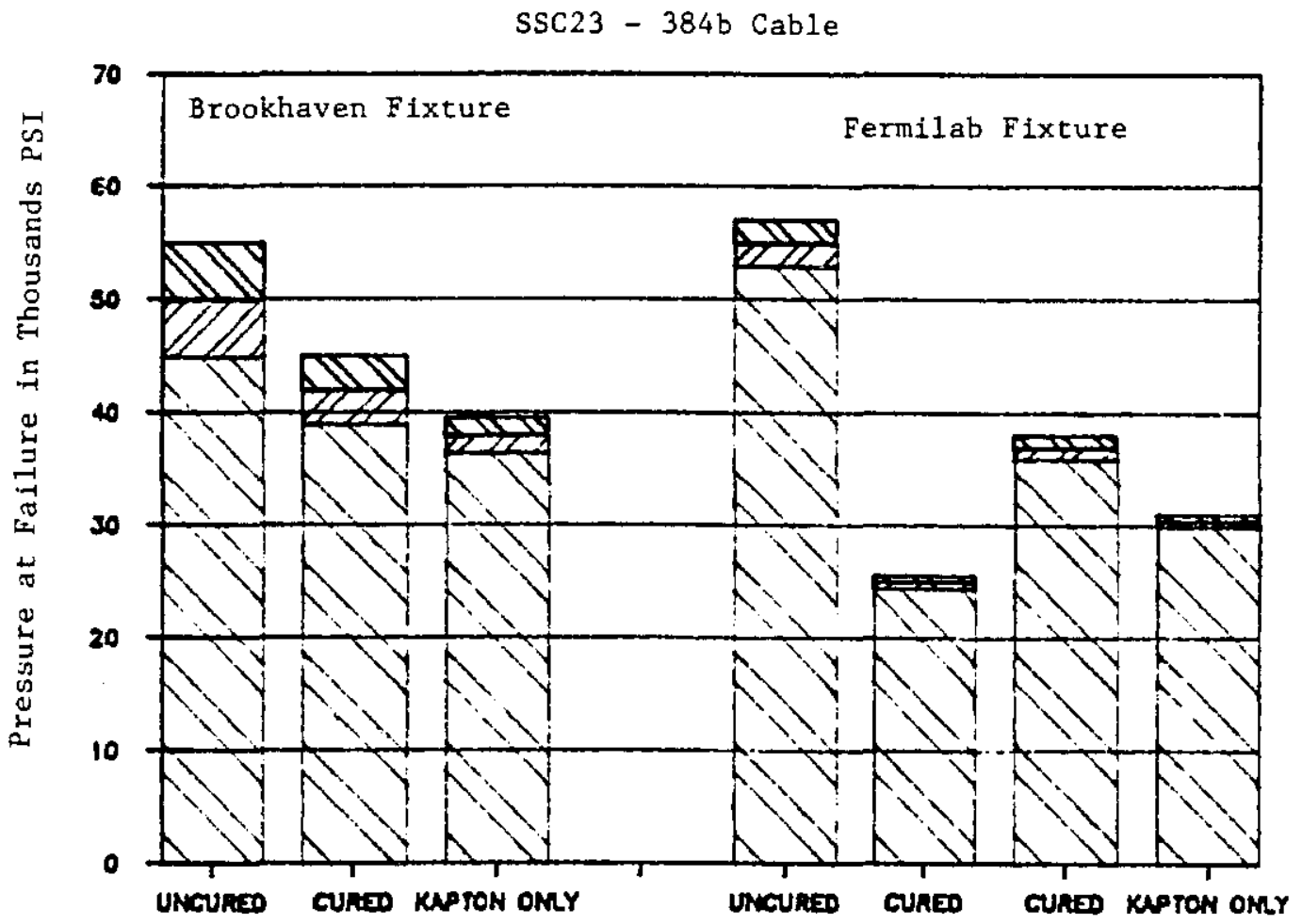

Fig. 8. Pressure to cause electrical breakdown of insulation consisting of Kapton only, or Kapton plus epoxy fiberglass, in both the cured and uncured state. 
The Brookhaven and Fermi fixtures are seen to give comparable results at pressure below the point where appreciable width expansion occurs. The cured epoxy-fiberglass was just slightly better than the Kapton only, which is to be expected since the epoxy does not form an integral film. The uncured epoxy-fiberglass samples withstood much higher pressures. We think that this is due to the lubricating effect of the uncured epoxy. The lubrication allows the layers of Kapton to slide past each other locally reducing the stresses in these places.

In order to be quite sure that our tests were giving the same pressure to breakdown that would be found in an actual coil, we molded a 3 inch long section of SSC outer coil. We loaded it in a curved fixture to simulate the collar and noted the pressures at which turn-to-turn shorts occurred. The results were that, with the exception of the one unusual short that was found right away (we did not test until $4 \mathrm{kpsi})$, the shorts began at $31 \mathrm{kpsi}$ and when the test stopped at $37 \mathrm{kpsi}, 10$ of the 19 turn-to-turn gaps were still unshorted. This is in very good agreement with the cable pair test data.

Figure 9 shows data from 8 different tests of Kapton only breakdown done with a section of SSC23-384b cable taken from the cable used to wind 4 different short coils. Each bar labeled $F$ is the average of 10 samples tested in the Fermi fixture, and the bars labeled B are the averages of 5 or 6 samples tested in the Brookhaven type fixture. The boxes at the top show the $+1-$ standard deviations. The numbers are coil numbers. Also shown are 3 tests of 5 samples each of new Kapton from DuPont in the same configuration of 1 layer of $1 / 2$ lapped Kapton only. The insulation was cut into $1 / 4^{\prime \prime}$ wide strips from sheets supplied by DuPont, and wrapped on the SSC23-384b cable by hand. The 100HA is .001" film of amorphous Kapton (the regular Kapton is crystalline), and the $100 \mathrm{MT}$ is the same film but filled with a powdered aluminum oxide, and the 130MT is the same filled film, but in a thickness of .130". The results of new and old film are very much the same.

Figure 10 shows the data from 6 different combinations of 2 layers of new Kapton each $1 / 2$ lapped. Also shown are data from two tests of old Kapton in the same two layer 1/2 lapped configuration. One of these is labeled Kapt.epo.cured., and was made from film which had been coated with a $.0001^{\prime \prime}$ layer of a $3 \mathrm{Mi}$ 's adhesive. This sample was cured before testing. This epoxy coated Kapton is the same that is being used to make our low beta quads. As in preceding figures, this one also shows the average of 5 tests as a single bar. All of these tests give averages in the range of $45 \mathrm{kpsi}$ to $55 \mathrm{kpsi}$ which is considerably higher than the $30 \mathrm{kpsi}$ to $38 \mathrm{kpsi}$ found for the single thickness of Kapton, but they do not show much difference from one film type to the other. All of the data of Figures 10 and 11 were obtained in the Brookhaven type fixture which restrains the cable from widthwise expansion.

DuPont has made the new Kapton films coated with a thermoplastic polymide adhesive and Brookhaven has wound some 3 foot coils from these films. We have cut and polished a section from one of these coils supplied to us by Brookhaven. The adhesive was a .0002" layer on either side of the film and the cables were wound with 2 layers $1 / 2$ lapped. Thus there are 8 layers of adhesive between each cable for a total thickness of $.0016^{\prime \prime}$. With that much adhesive, the 5000 kpsi pressure and $225^{\circ} \mathrm{C}$ temperature caused the adhesive to flow into the void space between cable strands pushing the Kapton layers with it. Photomicrographs of cross-sections of the Brookhaven coil and an SSC coil and one of our low beta quads have been made. They show the varying degrees to which the insulation systems fill the void between cable strands. The Brookhaven-new-Kapton-thermoplastic adhesive coil shows the coil completely filled, the SSC coil shows a void only partly filled, and the low beta quad shows a completely empty void space. It is presently uncertain what effect this has on coil performance. It should be noted that future Brookhaven coils may use less adhesive.

It has been noted that filling in the void between cable strands on the cable surface might increase the breakdown pressure by better distributing the loads. This effect might be seen in Skaritka's study of these new DuPont adhesive coated films. We have looked at this effect in two ways. For the first way, we have filled in the surface spaces between strands with a cured epoxy, and then wrapped the cable with Kapton with the usual 1 layer $1 / 2$ lapped and tested it in the Brookhaven type fixture which restrains widthwise expansion. On our second attempt to completely fill the surface with epoxy, three replications gave breakdown values ranging from 65 kpsi to $91 \mathrm{kpsi}$, or 2 to 3 times the breakdown with the same cable (SSC23-384b)! For the second 


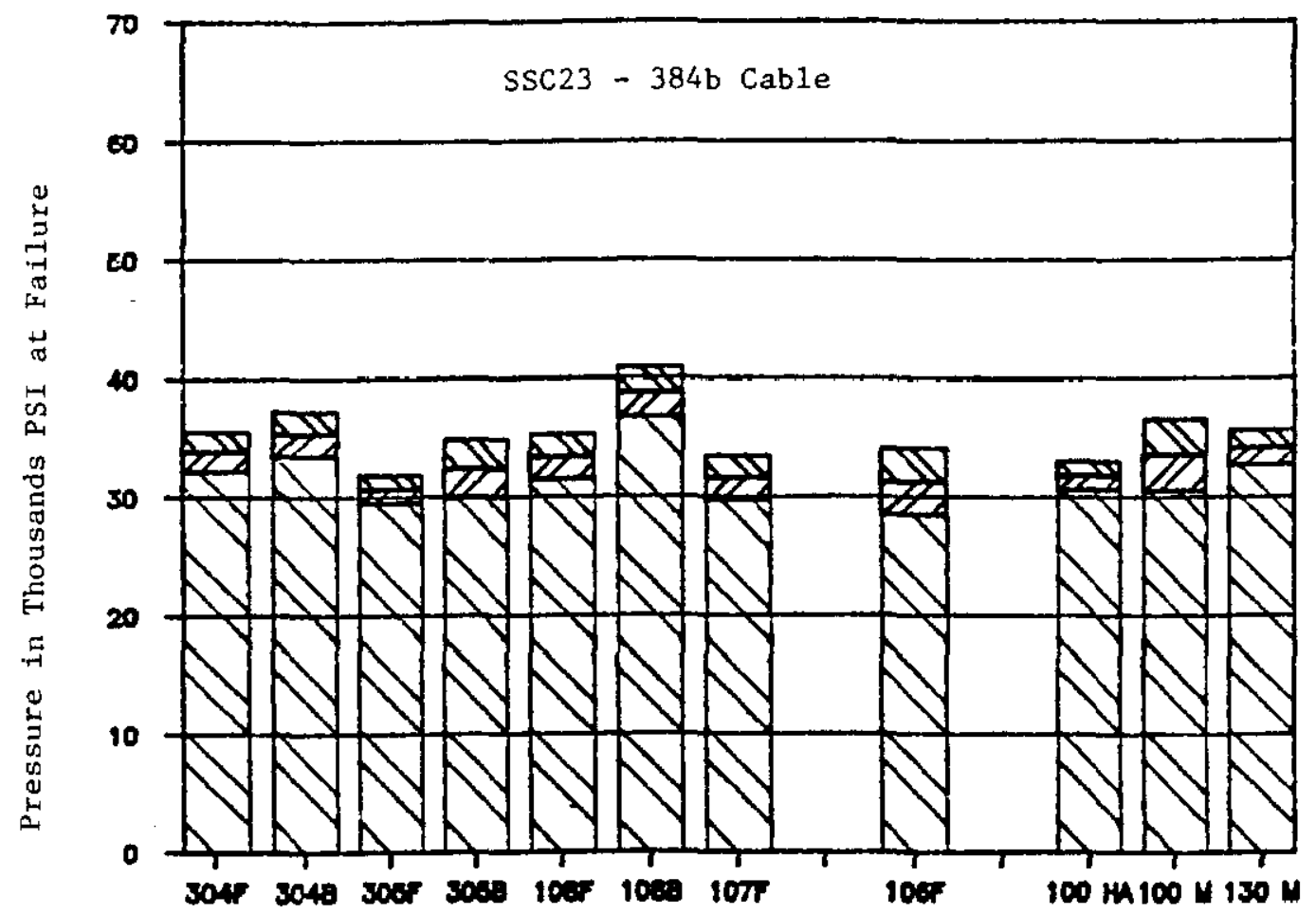

Fig. 9. Pressure to cause breakdown in a complete quality control series of SSC cable. Tests of new Kapton are also included.

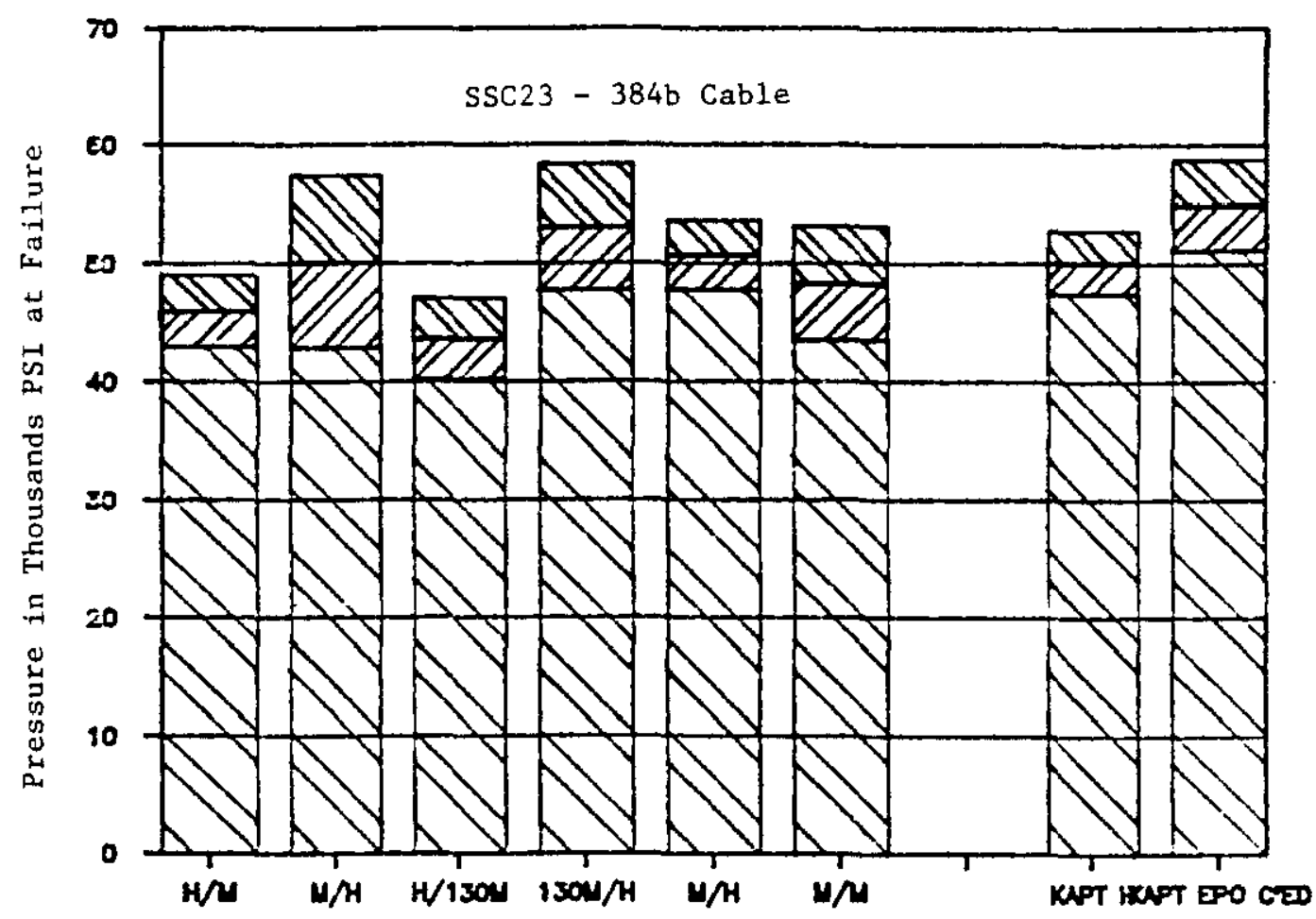

Fig. 10. Pressure to cause electrical breakdown in insulation consisting of 2 layers $1 / 2$ lapped of new Kapton, and also of old Kapton with and without cured epoxy adhesive. 
way, we took solid bars of copper of about the same cross-section as SSC cable except they were not tapered. We then had to test them in the Fermi type fixture with its parallel plates. Of course, we used the same insulation system as the epoxy filled cable. These 5 samples broke down at at an average value of $66 \pm 7 \mathrm{kpsi}$ (using the original unloaded area to calculate psi) and the failures were type 3; i.e., they failed when one of the copper bars expanded widthwise and tore the Kapton. We have not attempted to measure such pairs of copper bars in the Brookhaven fixture for fear of destroying our moderately hardened steel fixtures.

\section{ACKNOWLEDGEMENTS}

We are pleased to acknowledge the assistance of Choudet Khuon, Selles Morris, Daniel Rogers, Barbara Sizemore, Laurent Stadler, and Jim Cahill in taking and reducing the data.

\section{REFERENCES}

1. Ritchie, P.D., "Physics of Plastics," D. Van Nostrand Co., Princeton, NJ

2. Ward, I.M., "Mechanical Properties of Solid Polymers," John Wiley \& Sons Ltd., New York

3. Carson, J.A., and Markley, F.W., "Mechanical Properties of Superconducting Coils", IEEE Transactions on Magnetics, March, 1985, Volume MAG-21, \#2. A publication of the IEEE Magnetic Society, 345 East 47th Street, New York. 